\title{
Masonry buildings subjected to foundation settlements due to landslide: a preliminary study on the interpretation of structural behaviour using the load path method
}

\author{
F. Palmisano ${ }^{1}$ \& A. Elia ${ }^{2}$ \\ ${ }^{I}$ Department of Environmental Engineering and Sustainable \\ Development, Technical University of Bari, Italy \\ ${ }^{2}$ Department of Civil and Environmental Engineering, \\ Technical University of Bari, Italy
}

\begin{abstract}
Models have always been instruments to foresee the characteristics of a work. Only after Galilei, this custom entered the heritage of science and then models also become instruments to simulate and analyse structural behaviour. This paper reports the preliminary results of a study on the use of the load path method to interpret the behaviour of masonry buildings subjected to foundation settlements due to landslide. Models useful for describing global behaviour are presented. The aim is to show the effectiveness of the load path method for the prediction of structural behaviour, as well as for the diagnosis of crack patterns.
\end{abstract}

Keywords: masonry structures, landslide, strut-and-tie model, load path method.

\section{Load path method: basic principles}

Born as a method to design Strut-and-Tie Models (Schlaich and Schafer [1]), in reinforced concrete structures, the load path method (LPM) has also become a simple and effective instrument to understand the behaviour of masonry structures (De Tommasi et al. [2], Palmisano et al. [3]). It is not only a numerical method, but also a geometrical method that predicts calculation results disclosing the shape aspects from which it is possible to recognise real structural behaviour.

In the transfer of forces within a structure or an element, from their point of origin $(S)$ to their ends $(E)$, deviations in the load path direction can occur 


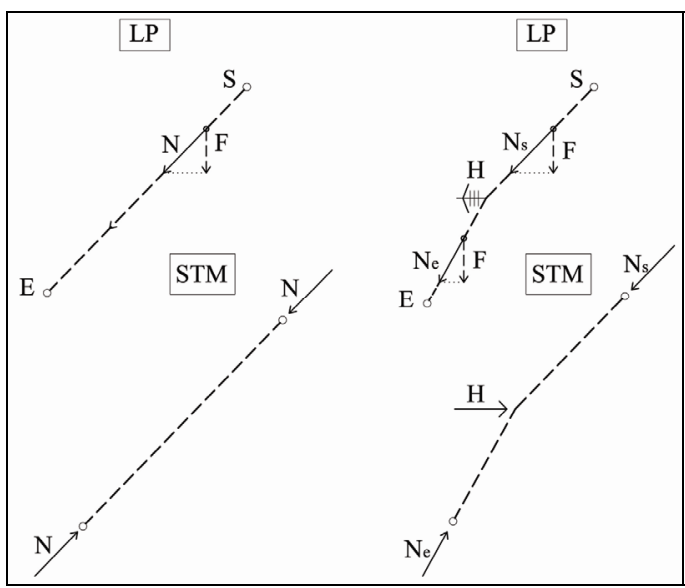

Figure 1: $\quad$ Load path (LP) and Strut-and-Tie Model (STM).

causing a thrust $(H)$; for equilibrium to be maintained, a reactive force must be applied that is equal in magnitude and opposite in direction to this thrust (fig. 1).

The load path (for further details on the basic principles of the method see references [2-4]) represents the line along which a force or a force component (more precisely: the component of a force in a chosen direction, e.g. the vertical component of a load) is carried through a structure from the point of loading to its support. The force component ( $F$ in fig. 1) associated with a load path remains constant on its way through the structure; as a consequence of this definition, thrust $H$ must be perpendicular to the travelling load $F$. The design of this load flowing through the structure can be approximated by polygonal lines in which there are thrusts in every deviation node. Structure will be crossed by fluxes in compression (dashed lines, fig. 1), when loads travel in the same direction of their path, and by fluxes in tension (continuous lines, fig. 1) along which loads go in the opposite direction respect to their path. According to the classical theory, the basic principles of load path method are the respect of equilibrium and consistency. Thrusts in deviation nodes are necessary in order to respect equilibrium and every path is possible if it is in equilibrium.

Among infinite paths in equilibrium, loads have to choose the one in which their vectors invest the minimum quantity of strain energy $(D)$, that is the only one consistent and in equilibrium. At this purpose, loads get energy from their own potential energy that decreases.

The total invested strain energy is

$$
D=\frac{1}{2} \int_{V} \boldsymbol{\sigma} \varepsilon d V
$$

where $V$ is the integration domain, $\sigma$ and $\varepsilon$ are the stress and the strain vector respectively.

Along a generic path (polygonal in this model), the calculus of the invested strain energy $(D)$ is simplified in the summation of the terms relative to each side of the truss: 


$$
D=\sum_{i} D_{i}
$$

where $i$ is the generic side of the load path.

For instance, if linear elastic constitute laws for materials are assumed as well as constant transversal section of each side, the elementary strain energy $D_{i}$ is

$$
D_{i}=\frac{1}{2} N_{i} l_{i} \varepsilon_{i}
$$

where $i$ is the generic side of the load path, $N_{i}$ is the intensity of the vector that bears the travelling load on that side, $l_{i}$ is the length of the generic side and $\varepsilon_{i}$ is the mean strain on that side.

If, in the assumption of linear elastic constitute laws for materials with Young Modulus equal to $E$, the transversal section of a side is linearly variable from $A^{(I)}{ }_{i}$ to $A^{(2)}{ }_{i}$, the elementary strain energy $D_{i}$ is

$$
D_{i}=\frac{1}{2} \frac{N_{i}^{2} l_{i}}{E\left(A_{i}^{(1)}-A_{i}^{(2)}\right)} \operatorname{Ln}\left(\frac{A_{i}^{(1)}}{A_{i}^{(2)}}\right)
$$

\section{Behaviour of a masonry panel subjected to foundation settlements}

In the macro-modelling approach, there is no distinction between bricks and mortar joint because these elements are taken into account by a homogenisation criterion. In this paragraph, the analysis of a masonry panel subjected to foundation settlements due to landslide is performed using the load path method.

For the sake of shortness, only the case of a settlement that involves the lateral part of a masonry pane is considered.

The following assumptions have been made:

- settlement is only due to the landslide movement; it does not depend on the loads acting on the masonry panel;

- masses are concentrated;

- the masonry panel is infinitely stiff respect to the foundation soil;

- a perfectly plastic constitutive law at the ultimate limit state has been hypothesised for the soil.

Despite these assumptions, that benefit the simplicity of the analysis, the method has general validity.

Fig. 2 shows the structure at the state 1 (static equilibrium before the soil settlement). In this state, because of the simplicity of the model, there are only descending loads and, in this macro-model, there are no deviations of the travelling loads. Actually, a microscopic analysis would show that travelling loads have to deviate in order to cross the brick-mortar interface (De Tommasi et al. [2]).

In figs. 3-6, four different and possible (i.e. in equilibrium) load paths at the state 2 (after the soil settlement) are represented. For the sake of simplicity, the soil settlement has been modelled as a complete loss of contact with the right bottom side of the masonry panel. 


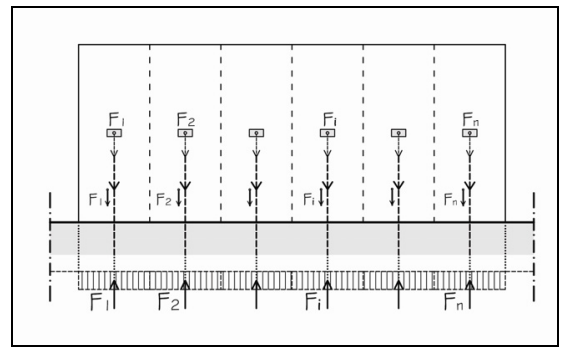

Figure 2: $\quad$ State 1: static equilibrium condition before the soil settlement.

Such loss of contact at the soil-panel interface causes the interruption of some load paths of the state 1 , the relevant modification of the interrupted paths and the modification of other paths in order to restore the global equilibrium condition. It is worth noting that, differently from what shown in figs. 3, 4, 5 and 6 , the components $F_{i}$ should divide themselves into parts which should follow different paths in order to minimise the total strain energy of the system. Nonetheless, a simplified sketch, representing the whole load which follows a chosen morphology of path, has been reported in the figures. Such assumption seems in fact to be very useful to immediately catch the 'dominant path' (i.e. the one followed by a big part of the total load) in order to enlighten the failure mechanism and the relevant crack pattern.

The models in figs. 3, 4, 5 and 6 make easily catch the effects of the soil settlement on the structure:

- because of equilibrium conditions the soil pressure diagram in the state 2 is different from that of state 1; moreover, because of the adopted assumptions, the soil pressure is nil on an area symmetrical to the one that has undergone the settlement;

- paths in tension arise in the masonry panel;

- load deviations generate thrusts that can find equilibrium by paths in tension and in compression.

The analysis of state 2 is also useful to understand how a structural element can evolve towards following states. For instance in fig. 7 a simplified model of the generation of an arch-shaped crack is shown. According to the above mentioned, in state 2 part of the loads follow an inclined ascent path (fig. 7-a) in order to find a new equilibrated configuration. When the stress in an inclined path in tension reaches the tensile strength of the masonry panel (beginning of the state 3) the first crack is generated (fig. 7-b). The consequent bypass of this crack generates a concentration of tensile stresses around the fissure (because loads, in order to minimise strain energy, tend to follow shorter paths) that causes the extension of the crack up to its stabilisation (end of the state 3; fig. 7c). In this last phase, the part of the masonry panel underneath the crack finds again the contact with the soil and, consequently, its loads restart to follow a path similar to that of state 1 . On the other side, loads above the crack are now obliged to move out of the crack and then can cause further cracks at the top of the masonry panel. 


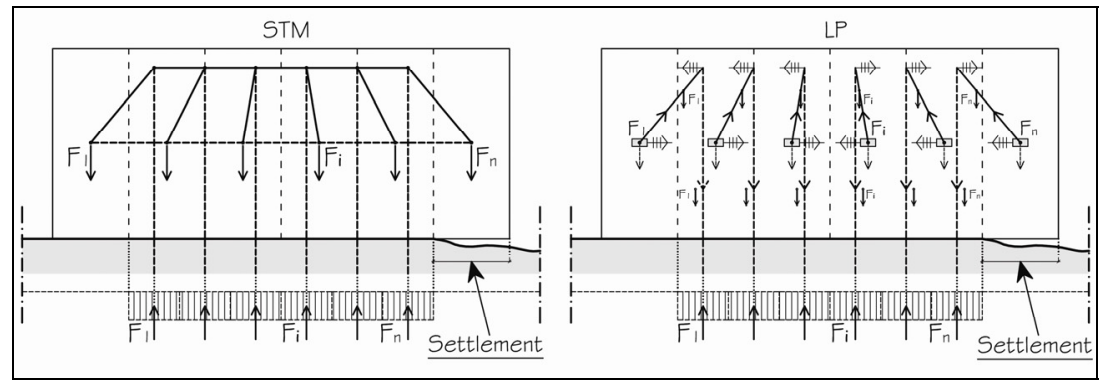

Figure 3: $\quad$ State 2: load path LP(1).

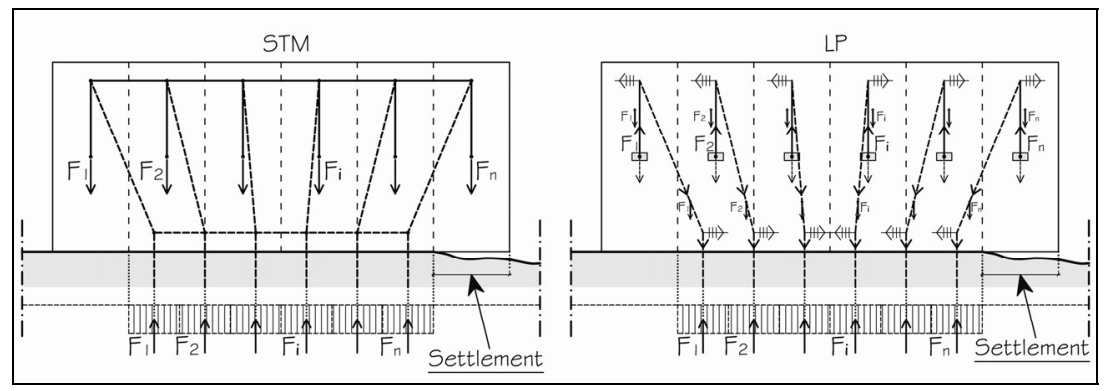

Figure 4: $\quad$ State 2: load path LP(2).

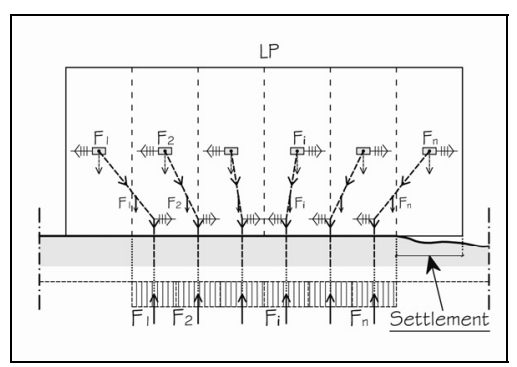

Figure 5: State 2: load path LP(3).

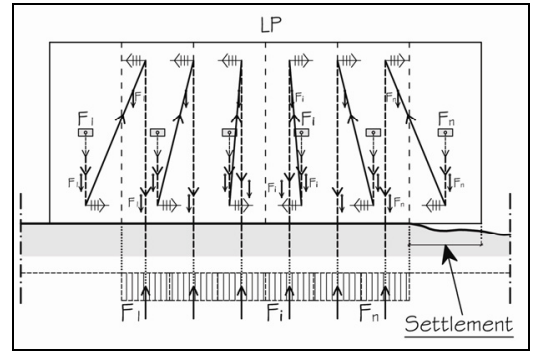

Figure 6: State 2: load path LP(4).

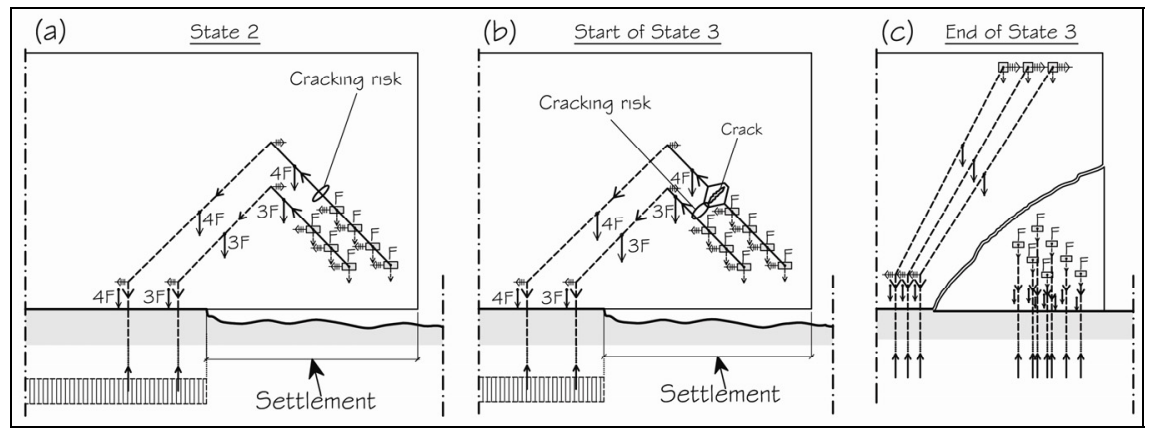

Figure 7: Formation of an arch-shaped crack due to soil settlement. 


\subsection{Energetic analysis}

In figs. 3-6 four different and possible (i.e. in equilibrium) load paths are shown. As above discussed (see the first paragraph), only one path respects the equilibrium conditions and, at the same time, minimise the total strain energy of the structural system. For complicated systems, the solution (i.e. the identification of this path) can be found either by FEM analysis or by optimisation algorithms. As shown in the previous paragraph, there are many cases in which it could be very useful to search the 'most plausible solution' instead of the exact solution. The 'most plausible solution' is the path that, among different equilibrated load paths, has the lowest value of the total strain energy. This approach can be carried out by very simple mathematical methods and, in many cases, seems to be very useful because it is able to immediately catch the 'dominant path' (i.e. the one followed by a big part of the total load) in order to highlight, immediately and easily, the failure mechanism and the relevant crack pattern.

In this paragraph, according to this simplified approach, the energetic analysis of the path in figs. 3, 4, 5 and 6 is discussed. The masonry panel is considered in the state 2 (i.e. after soil settlement but before crack formation).

In an orthogonal Cartesian system $\mathrm{X} \Omega \mathrm{Y}$ (where $\mathrm{X}$ is the horizontal axis, $\mathrm{Y}$ is the vertical axis and $\Omega$ is the origin in the middle of the base of the masonry panel; figs. 8 and 9) the following assumptions have been made:

- the masonry panel is geometrically symmetrical and symmetrically loaded;

- masonry is treated as a homogeneous material;

- the masonry panel is loaded by a uniform gravitational volume load.

Thanks to symmetry conditions, the analysis can be referred only to half panel. It is worth noting that, due to the assumptions in paragraph 2, the load path is symmetrical even though the settlement is only on the right side of the panel. In the following, $H, B=2 b, S$ and $\gamma$ are the total height, the width, the constant thickness and the unit weight of the masonry panel respectively.

Moreover:

- $\alpha=\rho \cdot H$ is the width of the soil settlement;

- $\omega=E_{c} / E_{s}$ is the ratio of the compression Young Modulus of the panel to the tension one;

- $x_{a}$ and $x_{b}$ are the mesh width in the $\mathrm{X}$ direction of the panel and of the reactive soil;

- $n$ is the mesh number of the semi-width $b$ of the panel.

In the following, only the calculus of the strain energy of LP(1) is shown; nonetheless, an analogous procedure can be used to calculate strain energy in the other cases.

The following parameters have been used in the analysis (fig. 9):

- $A_{\text {sup }, i}$ and $A_{\text {inf }, i}$ are the top and the bottom transversal area of the struts;

- $\theta_{i}$ is the inclination of the $i$ inclined strut;

- $A_{i}$ is the transversal area of the vertical strut;

- $\delta_{s}$ defines the position, along the Y direction, of the load deviation nodes in the top part of the panel; 


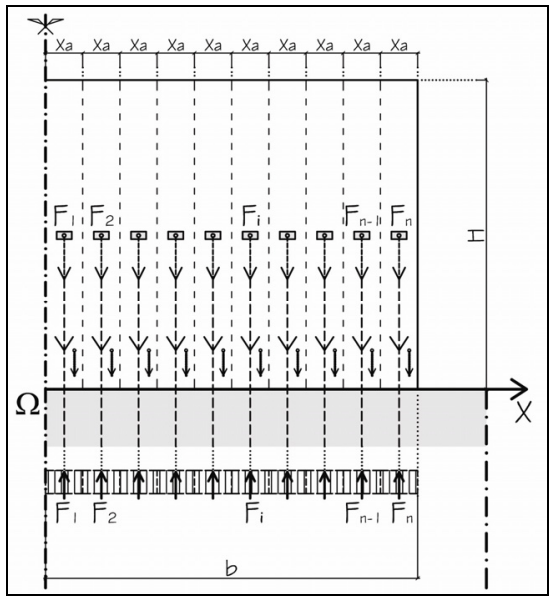

Figure 8: State 1: parameters to calculate total strain energy.

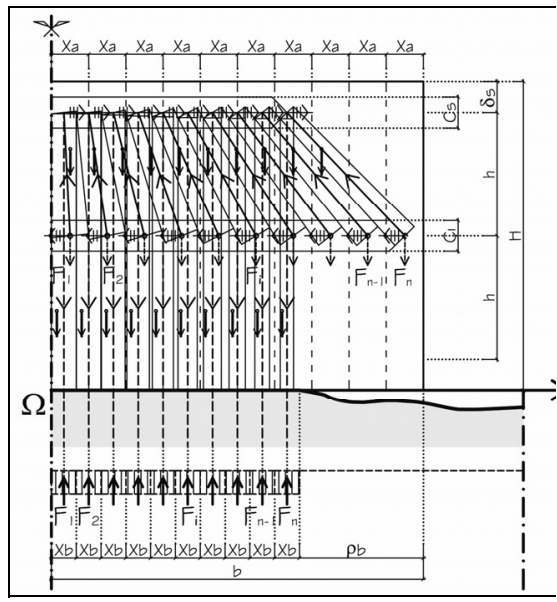

Figure 9: State 2: parameters to calculate total strain energy of LP(1).

- $h=H / 2-\delta_{s}$;

- $C_{s}$ and $C_{i}$ are the thickness of the top and of the bottom longitudinal boom assumed constant.

Strain energy has been calculated according to eqns. (1)-(3). The strain energy of the inclined ascent and of the vertical descent is respectively equal to

$$
D_{L P 1}^{(I)}=\frac{\omega}{2 E_{c}} \sum_{i=1}^{n}\left[\frac{N_{i}^{2} l_{i}}{\left(A_{\mathrm{sup}, i}-A_{\mathrm{inf}, i}\right)} \operatorname{Ln}\left(\frac{A_{\mathrm{sup}, i}}{A_{\mathrm{inf}, i}}\right)\right] ; D_{L P 1}^{(I I)}=\frac{H-\delta_{s}}{2 E_{c}} \sum_{i=1}^{n} \frac{\left(F_{i}\right)^{2}}{A_{i}}
$$

where

$$
N_{i}=\frac{F_{i}}{\sin \theta_{i}} ; l_{i}=\frac{h}{\sin \theta_{i}} ; \theta_{i}=\tan ^{-1}\left[\frac{2 h \cdot n}{\rho H(2 i-1)}\right]
$$

The strain energy of the top and bottom longitudinal boom is respectively equal to

$$
D_{L P 1}^{\text {(sup) }}=\frac{1}{2} \frac{\omega \cdot x_{b}}{E_{c} \cdot S \cdot C_{S}} \sum_{j=1}^{n}\left(\sum_{i=1}^{j} \frac{F_{i}}{\tan \theta_{i}}\right)^{2} ; D_{L P 1}^{\text {(inf) }}=\frac{1}{2} \frac{x_{a}}{E_{c} \cdot S \cdot C_{I}} \sum_{j=1}^{n}\left(\sum_{i=1}^{j} \frac{F_{i}}{\tan \theta_{i}}\right)^{2}
$$

where

$$
\theta_{i}=\tan ^{-1}\left[\frac{2 h \cdot n}{\rho H(2 i-1)}\right]
$$

Finally the total strain energy of $\operatorname{LP}(1)$ is

$$
D_{T O T}^{L P 1}=D_{L P 1}^{(I)}+D_{L P 1}^{(I I)}+D_{L P 1}^{(\text {(sup) }}+D_{L P 1}^{(\text {inf }}
$$

From the visual analysis of the paths in figs. 3-6, it can be deduced that combining the LP(1) with LP(3) there should be a saving in the strain energy, with respect to the shown paths, due to the absence of a thrust path in the middle of the panel (fig. 10). 


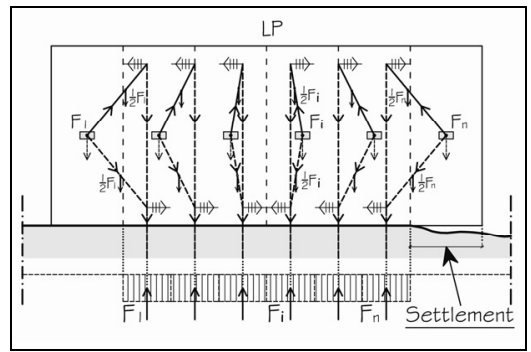

Figure 10: $\quad$ State 2: load path LP(5); combination of LP(1) with LP(3).

It is possible to introduce a 'reference strain energy' $D_{R E F}$ that is the strain energy invested by the total load of half panel to reach the foundation soil by a vertical direct path in compression (i.e. that invested at state 1 in fig. 8). In the examined case the reference strain energy is

$$
D_{R E F}=\frac{S b \gamma^{2} H^{3}}{4 E_{c}}
$$

In figs. 11 and 12 the ratio $D_{T O T} / D_{R E F}$ has been plotted for $\omega$ values equal to 1 and 3 respectively. To plot these diagrams the following assumptions have been made:

- $H=6 m ; b=9 m ; n=10$;

- for $\mathrm{LP}(1), \mathrm{LP}(2)$ and $\mathrm{LP}(4)$ the axis of the top boom is $H / 10$ distant from the top edge of the panel;

- for $\operatorname{LP}(2), \operatorname{LP}(3)$ and $\operatorname{LP}(4)$ the axis of the bottom boom is $H / 10$ distant from the bottom edge of the panel;

- the thickness of the booms has been assumed as the maximum value consistent with their positions without causing superimposition of the booms.

The ratio $D_{T O T} / D_{R E F}$ quantifies the increase of strain energy in the panel because of soil settlement and gives the opportunity to immediately catch which is the 'most plausible path' (i.e. the one with the minimum value of the total strain energy).

It is worth noting that in all the cases showed in figs. 11 and 12 , the $\operatorname{LP}(5)$ is the 'most plausible solution'. This is a direct consequence of the abovementioned consideration about the saving in the strain energy of this load path due to the elimination of the thrust path in the middle of the panel.

\section{Concluding remarks}

This paper has been proposed as a preliminary study concerning the use of LPM to interpret the behaviour of masonry buildings subjected to foundation settlements due to landslide. Models useful to understand global behaviour have been showed.

The aim of this work is to show that the load path method can be a versatile and effective instrument to study masonry structures because it seems to 


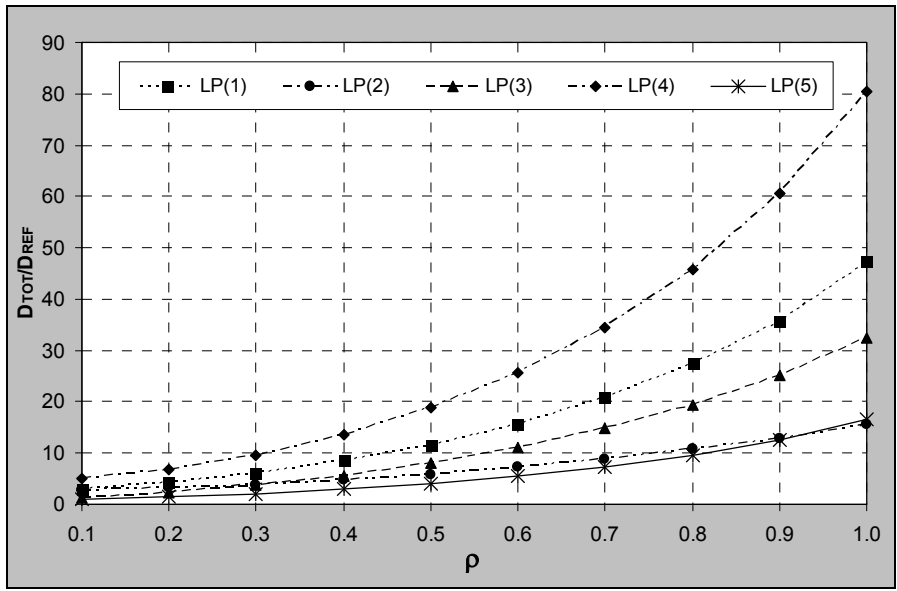

Figure 11: $\quad \mathrm{D}_{\mathrm{TOT}} / \mathrm{D}_{\mathrm{REF}}$ in the case of $\omega=1$.

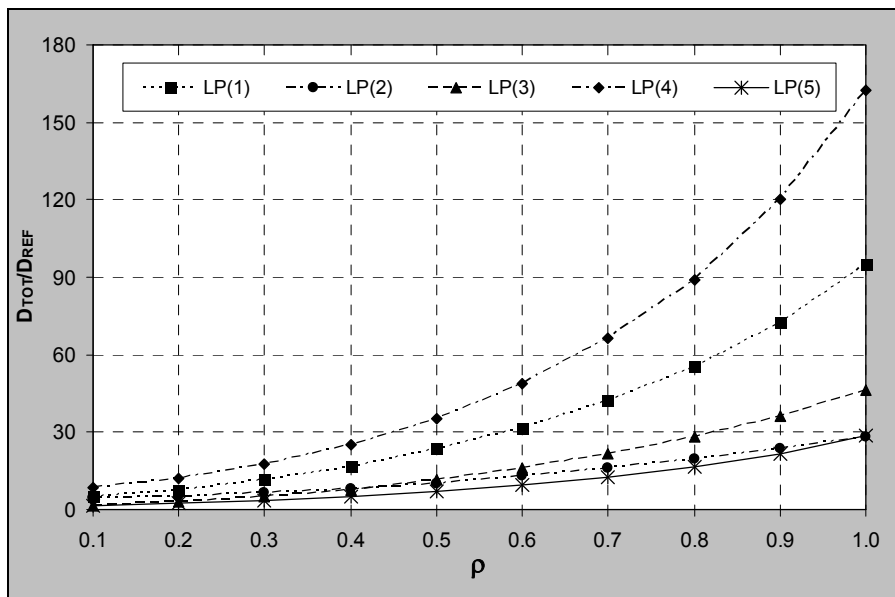

Figure 12: $\quad \mathrm{D}_{\mathrm{TOT}} / \mathrm{D}_{\mathrm{REF}}$ in the case of $\omega=3$.

conciliate successfully the necessity to get a numerical solution without losing touch with the perception of the synthesis of physical structural behaviour.

Further theoretical work is needed to apply the method to real cases and to compare the results with those obtained by other analyses (e.g. FEM).

\section{Acknowledgement}

The work presented in this paper was funded by Apulian Region under the Strategic Research Project n. 119 "Landslide risk assessment for the planning of small centres located in chain areas: the case of the Daunia region". 
150 Structural Studies, Repairs and Maintenance of Heritage Architecture XI

\section{References}

[1] Schlaich, J. \& Schafer, K., Designing and detailing using Strut-and-tie Models. Proc. of the Workshop Strut-and-Tie Models for the Design of Structural Concrete, ed. K. Shafer: Tainan, National Cheng Kung University, 1996.

[2] De Tommasi, G., Monaco, P. \& Vitone, C., A first approach to load path method on the masonry structures behaviour. Structural Studies, Repairs and Maintenance of Heritage Architecture VIII, ed. C.A. Brebbia, WIT Press: Southampton, United Kingdom, pp. 287-296, 2003

[3] Palmisano, F., Vitone, A., Vitone, C., Load path method in the interpretation of the masonry vault behaviour", Structural Studies, Repairs and Maintenance of Heritage Architecture IX, ed. C.A. Brebbia \& A. Torpiano, WIT Press: Southampton, United Kingdom, pp. 155-167, 2005.

[4] Palmisano, F., Vitone, A., Vitone, C., Il metodo del percorso del carico. Teoria e pratica delle costruzioni in cemento armato. Volume II - La progettazione esecutiva e la realizzazione, eds. M. Mezzina, A. Vitone, D. Raffaele, Città Studi Edizioni (De Agostini Scuola SpA): Novara, Italy, pp. 3-38, 2007. 